\title{
The Close Binary Frequency of Wolf-Rayet Stars as a Function of Metallicity in M31 and M33
}

\author{
Kathryn F. Neugent and Philip Massey \\ Lowell Observatory \\ emails: KNeugent@lowell.edu, massey@lowell.edu
}

\begin{abstract}
Here we investigate whether the inability of the Geneva evolutionary models to predict a large enough WC/WN ratio at high metallicities (while succeeding at lower metallicities) is due to their single star nature. We hypothesize that Roche-lobe overflow in close binary systems may produce a greater number of WC stars at higher metallicities. But, this would suggest that the frequency of close massive binaries is metallicity dependent. We now present our results based on observations of $\sim 100$ Wolf-Rayet binaries in the varying metallicity environments of M31 and M33.
\end{abstract}

Keywords. stars: Wolf-Rayet, binaries: close, stars: evolution

Massive star evolutionary models generally predict the correct relative number of WCtype and WN-type Wolf-Rayet (WR) stars at low metallicities, but underestimate the ratio at higher (solar and above) metallicities (Meynet \& Maeder 2005; Neugent et al. 2012). One possible explanation for this failure is perhaps single-star models are not sufficient and Roche-lobe overflow in close binaries is necessary to produce the "extra" WC stars at higher metallicities. However, this would require the frequency of close massive binaries to be metallicity dependent. Here we test this hypothesis by searching for close WR binaries in the high metallicity environments of M31 and the center of M33 as well as in the lower metallicity environments of the middle and outer regions of M33.

To identify the relative frequency of WR binaries in M31 and M33, we observed 250 candidates 4-6 times across a period of three months. We then looked for stars with statistically significant radial velocity variations by comparing the internal errors $(I)$ with the external scatter $(E)$. Stars with large external radial velocity scatter relative to a small internal error are likely to be close binaries. To account for underestimated internal errors, we adopted an $E / I$ value of $>2$ as the dividing line between close binaries and non-binaries (Abt \& Levy 1976; Abt 1987). An example of a star with a relatively high $E / I$ value of 4.4 is shown below. Note that the emission comes from the WR (WN5) while the absorption comes from an OB companion (B0).

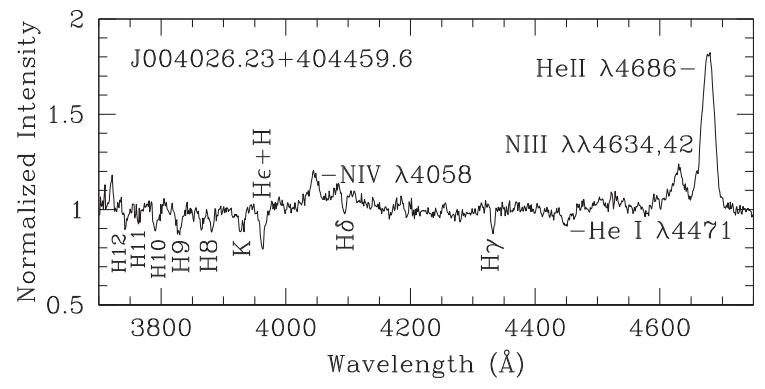


The table below shows the frequency of binaries detected among the M31 and M33 WRs, where we've broken M33 up into three separate regions based on its varying metallicity (see Neugent \& Massey 2011). The percentages of close binaries agree across all regions to within a few percent, with the exception of the inner portion of M33. In this region we find that the fraction of close binaries is lower than expected, so this is in the opposite sense of what we would expect if binarity was responsible for the overabundance of WCs. Additionally, we find that the data are consistent with the close binary frequency of WC stars being essentially identical in all four regions.

\begin{tabular}{lccccc} 
Region & $\log \frac{O}{H}+12$ & Total & \% Binary & \multicolumn{2}{c}{ \% Binary } \\
& & \# Stars & $\frac{E}{I}>2$ & WNs & WCs \\
\hline M31 (all) & 8.9 & 106 & $44 \pm 8$ & $57 \pm 12$ & $27 \pm 9$ \\
M33 $(\rho<0.25)$ & 8.7 & 44 & $23 \pm 8$ & $20 \pm 10$ & $26 \pm 13$ \\
M33 $(0.25 \leqslant \rho<0.50)$ & 8.4 & 46 & $46 \pm 12$ & $47 \pm 14$ & $40 \pm 24$ \\
M33 $(\rho \geqslant 0.5)$ & 8.3 & 54 & $44 \pm 11$ & $43 \pm 12$ & $50 \pm 27$
\end{tabular}

While studying the relationship between binary frequency and metallicity, we also investigated the nature of our 102 newly identified close WR binaries. With a maximum of 9 observations per star, determining a period was nearly impossible, as shown below where we have phased the radial velocity measurements of J013402.93+305126 by a strongly indicated period of 2.648 days. Clearly, many more observations are needed. Luckily, we have time scheduled on Lowell Observatory's Discovery Channel Telescope this fall to photometrically monitor the most promising binaries to determine periods. Then, we hope to calculate orbits and, finally, masses.

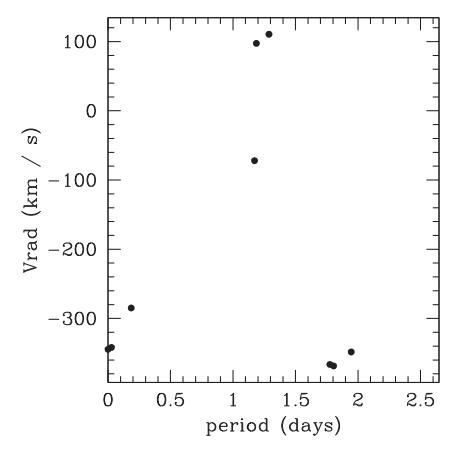

Out of the $250 \mathrm{WRs}$ in M31 and M33, we found 102 stars with $E / I>2$. These close binaries are found throughout M31 and M33, with a binary frequency independent of location, except in the center of M33 where fewer WN binaries were found. Thus, we conclude that a larger binary frequency at high metallicities is not responsible for the discrepancy with the models. Instead, the models seem to either underestimate the life spans of WCs and/or overestimate the life spans for WNs at high metallicities.

Acknowledgements. This work was supported by the NSF under AST-1008020.

\section{References}

Abt, H. A. 1987, ApJ 317, 353

Abt, H. A. \& Levy, S. G. 1976, ApJS 30, 273

Meynet, G. \& Maeder, A. 2005, A\&A 429, 581

Neugent, K. F. \& Massey, P. 2011, ApJ 733, 123

Neugent, K. F., Massey, P., \& Georgy, C. 2012, ApJ 759, 11 